\title{
Alberto Casadei, Dante oltre l'allegoria
}

\author{
Ravenna, Longo, 2021, pp. 272, € 24
}

In un anno di celebrazioni dantesche, "carco" di pubblicazioni specialistiche e divulgative dedicate al poeta e alla sua opera, si assiste spesso alla ripetizione di acquisizioni critiche e filologiche e alla sostanziale fedeltà a interpretazioni e idee di illustri studiosi del passato più o meno recente. Non è questo lo spirito che anima la recente pubblicazione, presso l'editrice Longo, della monografia di Alberto Casadei dal titolo Dante oltre l'allegoria. Volume di taglio specialistico, uscito a pochi mesi dall'agile libro divulgato comparso in libreria nel 2020 per il Saggiatore (dove è illustrata una proposta cronologica per le opere di Dante), Oltre l'allegoria raccoglie i risultati di ricerche svolte a partire dal 2009 (alcuni capitoli avevano già visto la luce in diversa forma su rivista), mirando a indicare una nuova chiave di lettura per la Commedia che vada oltre la categoria dell'allegoresi, perfettamente espressa nell'ultimo capitolo prima dell'appendice.

Per parlare di questo libro occorre forse cominciare proprio dal capitolo 7 (Dante oggi) e dalla nuova proposta interpretativa di Casadei per il testo della Commedia, che mira in prima istanza ad un'analisi narrativa "che non esclude, ma nemmeno è determinata dalle componenti allegoriche» (p.165), in grado di giustificare le 'finzioni' del racconto e le varie ipotesi storico-culturali, intercettandone le costanti antropologiche manifestate nelle varie forme di racconto lungo tutto il percorso narrativo dantesco. Ecco quindi spiegato il titolo del volume, precisando che Casadei non rinnega il valore di allegoria e allegoresi come "strumenti ermeneutici utilizzabili», ma non vuole nemmeno renderli «vincoli necessari» alla comprensione del testo dantesco. Ripercorrendo nel capitolo e sotto questa nuova luce ermeneutica anche idee già prospettate nei precedenti, la proposta interpretativa di Casadei si esplica in una complessiva disanima non solo di alcuni punti nevralgici del poema, ma anche di tutto il percorso letterario e biografico di Dante, arrivando quindi a avanzare un'interpretazione per il XXI secolo che, senza rinnegare la grande letteratura critica sull'argomento (che lo studioso maneggia con invidiabile perizia), adatti al nostro immaginario "laico e desacralizzato» la tensione dantesca al "supremo» (p. 203). Con questo spirito nella prima parte del volume, Ancora accertamenti e punti critici (esplicito richiamo al libro del 2019), si analizza nuovamente la validità della testimonianza di Boccaccio sulla composizione fiorentina dei 
primi sette canti, accertando la possibile buona fede del Certaldese e la sua affidabilità nella sostanza. L'analisi di Casadei è tutta basata sui testi, in particolare l'epistola IV e la canzone Montanina, riletti e interpretati sulla base del forte distacco stilistico tra il IV e il V dell'Inferno e su alcune problematiche incongruenze tra i primi canti infernali e la dottrina esplicitata nel Convivio (specialmente nel IV libro), senza mai nascondere comunque in nota le idee avverse alle sue o i punti che potrebbero rendere la sua ricostruzione problematica. Una definizione ermeneutica che passa anche attraverso l'analisi di vari modi utilizzati a partire dall'Ottocento per leggere il poema dantesco, esposti nella seconda parte: Tempi e modi della ricezione dantesca. Qui sono affrontate sia le interpretazioni del poema di illustri studiosi, da Domenico Mauro a Giovanni Pascoli, portatori di due differenti modi di leggere la Commedia sempre all'ombra dell'allegoria o di una lettura metaforico simbolica, sia le illustrazioni moderne della Commedia, veicoli di una esegesi e comprensione del testo, ma da esso sempre più autonome, all'interno delle quali si possono osservare «elementi che appartengono più all'intuizione di tratti subliminari, addirittura biologico-cognitivi e trans-storici» (p. 148) ancora colti da lettori lontani dalle originali coordinate cronotopiche di Dante (facendo uso anche delle teorie sviluppate nel volume Biologia della Letteratura del 2018). Da qui la forza delle illustrazioni della Commedia, in grado di ri-creare, quasi sostituire, l'originale testo dantesco e di utilizzarlo per spiegare la contemporaneità, rivitalizzando il testo. Da queste coordinate trae origine la "giustificazione" dello studioso per la proposta di lettura della Commedia oggi, un'interpretazione che verrà certamente discussa in ambito specialistico nei prossimi anni e che ha il merito oggettivo di non volersi accontentare di quanto espresso fino a oggi dagli studi.

Un analogo discorso organico merita l'appendice del libro, che in sette note dantesche vuole proporre alcune soluzioni esegetiche di grande impatto. I luoghi analizzati da Casadei sono infatti tra i più discussi e problematici per la critica dantesca: dal "Durante" del Fiore fino all'interpretazione dell'endecasillabo di Par. XXXI, passando per la cronologia delle quindici canzoni dantesche ad alcune note sui commenti trecenteschi alla Commedia. L'intento di Casadei è quello di proporre nuove interpretazioni, avanzando l'ipotesi più ragionevole rispetto ai dati in nostro possesso, presentando soluzioni esegetiche destinate certamente ad essere molto discusse nel prossimo periodo. Solo per fare qualche breve cenno: nella nota 3, viene proposta una convincente datazione dei due congedi conosciuti della canzone Tre Donne, in grado di tenere insieme la complessa cronologia storico-politica che vedrebbe dei cambiamenti radicali negli atteggiamenti tenuti dal poeta a seguito della disastrosa battaglia della Lastra. La questione non è certo oziosa, essendo ben evidente (e già espresso nel "corpo principale" del libro) il cambiamento di Dante nel corso dei primi anni del Trecento, legato alla stesura del Convivio, e la datazione qui offerta della canzone potrebbe aprire interessanti orizzonti esegetici anche nella visione generale dell'atteggiamento dantesco rispetto agli altri testi coevi e alle possibili 
redazioni autoriali. Una sezione la cui lettura difficilmente lascerà lo studioso di Dante neutro di fronte alle soluzioni proposte.

In conclusione, un volume complesso e articolato, che pur rivolgendosi a un pubblico specialistico dialoga con i recenti saggi divulgativi dell'autore ed è dotato di un'unità contenutistica molto più marcata di quanto si potrebbe pensare sfogliando l'indice. Casadei riesce quindi a avanzare una personale interpretazione del "più bel libro scritto dagli uomini", ripercorrendo i maggiori studi sul testo e applicando il corredo teorico-critico da lui sviluppato nel corso di più di un decennio e qui perfettamente spiegato.

Valerio Cellai Università di Pisa 
